DOI: 10.12957/demetra.2018.31559

\title{
Triagem nutricional e riscos cardiometabólicos nos funcionários de um Restaurante Universitário
}

\section{Nutritional screening and cardiometabolic risks in the workers of a University Restaurant}

\author{
Ana Karina Furtado Mendes' \\ Anne Karollyne Cavalcante Carvalho' \\ Monalyza Guerra Justino' \\ Lucia Helena Almeida Gratão \\ Renata Junqueira Pereira' \\ 1 Universidade Federal do Tocantins, Curso de \\ Nutrição. Palmas, Tocantins, Brasil. \\ Correspondência / Correspondence \\ Renata Junqueira Pereira \\ E-mail: renatajunqueira@mail.uft.edu.br
}

\section{Resumo}

Introdução: Os riscos cardiometabólicos são caracterizados por um conjunto de disfunções no metabolismo que predispõem a eventos cardiovasculares, quando coexistem no organismo. Objetivo: realizar triagem nutricional e investigar fatores de riscos cardiometabólicos em funcionários de um Restaurante Universitário. Método: foi realizada uma coleta de informações de 18 funcionários, por meio de uma ficha de atendimento e os dados obtidos foram analisados através do software DietBox e tabelados pelo programa Microsoft Office Excel. Resultados: Dentre os participantes $33 \%$ apresentaram sobrepeso e maior risco para desenvolvimento de doenças crônicas não transmissíveis, sendo que os homens apresentaram maior porcentagem de perímetro abdominal (42,8\%). Além disso, foi possível notar que 33,3\% das pessoas estudadas apresentaram valores de HDL-c baixo e 27,7\% colesterol total aumentado. Em média, observaram-se variações do consumo diário de calorias em relação às recomendações de $38,8 \%$ para mais e $55,5 \%$ para menos. Em relação aos inquéritos alimentares, dentre os macronutrientes, destacou-se maior ingestão proteica e, dentre os micronutrientes, o sódio e selênio foram consumidos acima do recomendado e as fibras, enquanto cálcio e vitaminas $\mathrm{D}$ e $\mathrm{E}$ foram consumidos abaixo da recomendação. Conclusão: O estudo demonstrou a presença de fatores que predispõem a eventos cardiovasculares na população. Os aumentados índices de sobrepeso e obesidade nos indivíduos analisados foram possivelmente devidos a alterações dos padrões alimentares e reduzida da prática de atividade física.

Palavras-chave: Doenças Cardiovasculares. Dislipidemias. Sobrepeso. Deficiências Nutricionais. 


\section{Abstract}

Introduction: Cardiometabolic risks are characterized by a set of metabolic dysfunctions that predispose to cardiovascular events, when they coexist in the body. Objective: The purposes of this study are to perform the nutritional assessment and to investigate the cardiometabolic risk factors in the employees of a University Restaurant. Method: There have been collected socioeconomic, individual, anthropometric, alimentary data and biochemical exams (lipid profile and fasting glycemia) from eighteen employees through a data sheet and the data obtained were analyzed by the DietBox software. Results: Among the participants, 33\% were overweight and had higher risk of developing chronic noncommunicable diseases, where men presented higher percentage of abdominal circumference $(42.8 \%)$. Furthermore, it was possible to notice that $33.3 \%$ of the employees presented low HDL-C values and $27.7 \%$ increased total cholesterol. On average, daily calorie consumption variations were observed in relation to the recommendations of around $38.8 \%$ for more and $55.5 \%$ for less. Regarding feeding habit surveys, higher protein intake was observed and, among the macronutrients, it was noticed that the general daily intake values were according to the recommendations. Cholesterol, trans fat, sodium, selenium and vitamin C stood out being consumed over the expected; and fibers, calcium, magnesium and vitamins $\mathrm{D}$ and $\mathrm{E}$ were under the recommendation values. Conclusion: This study demonstrated the presence of factors that predispose cardiovascular events in the population. The high rates of overweight and obesity in the analyzed individuals were possibly due to changes in eating patterns and lack of physical exercises.

Keywords: Cardiovascular Diseases. Dyslipidemias. Overweight. Deficiency Diseases.

\section{Introdução}

De acordo com o National Cholesterol Education Program (NCEP), ${ }^{1}$ os riscos cardiometabólicos (RC) se caracterizam por um conjunto de disfunções no metabolismo e presença de fatores que predispõem a eventos cardiovasculares. Para avaliação desses fatores, são considerados a glicemia de jejum, as dosagens de triacilgliceróis e de HDL-colesterol, a aferição da pressão arterial e as variáveis antropométricas (circunferência abdominal, peso e altura), não se exigindo a comprovação da resistência à insulina. ${ }^{2}$ 
A morbimortalidade cardiovascular é observada tanto nos países desenvolvidos, quanto nos em desenvolvimento. ${ }^{2}$ Tal fato é resultado de alterações do estilo de vida, advindas dos processos de industrialização e urbanização, as quais têm atingido todas as raças, grupos étnicos e culturais. ${ }^{3}$

Um estudo epidemiológico com moradores de uma área rural de Santa Rosa - RS, na abrangência da Estratégia Saúde da Família, constatou que a prevalência de síndrome metabólica é de $8,5 \%$ em homens e $22 \%$ em mulheres, sendo mais elevada com o aumento da idade e com forte relação no sexo feminino. ${ }^{4}$

Analisando padrões alimentares de indivíduos com RC, Baxter ${ }^{5}$ observou que o consumo de dietas ricas em carnes gordurosas e grãos refinados (açúcares, trigo, sal) provocaram aumento da incidência de síndrome metabólica; enquanto dietas ricas em frutas, vegetais e grãos integrais foram correlacionadas negativamente à ocorrência da síndrome, sendo, inclusive, uma estratégia de prevenção e de tratamento da síndrome. ${ }^{6}$

Assim, o tratamento da síndrome metabólica inclui a associação de mudanças no estilo de vida com intervenções dietéticas, prática de atividade física regular e uso de fármacos. ${ }^{7,8}$ Desse modo, esta associação promove uma redução expressiva na circunferência abdominal, gordura visceral, pressão arterial, níveis de triglicérides e aumenta de forma considerável o HDL-colesterol. ${ }^{2,8}$

O objetivo desse estudo foi realizar triagem nutricional e investigar fatores de riscos cardiometabólicos em funcionários do Restaurante Universitário da Universidade Federal do Tocantins, por meio de atendimentos ambulatoriais, dosagens bioquímicas, avaliação do consumo alimentar e anamnese para coleta de dados sociodemográficos, de saúde e de estilo de vida.

\section{Metodologia}

Trata-se de um estudo transversal que teve como base populacional 33 funcionários do Restaurante Universitário (RU), da Universidade Federal do Tocantins, Campus de Palmas, sendo que apenas 18 aceitaram participar e assinaram o Termo de Consentimento Livre Esclarecido (TCLE).

Os dados obtidos por meio do recordatório $24 \mathrm{~h}$ (R24h) e a prescrição dietética foram gerados utilizando o software DietBox, calculando os nutrientes a partir da tabela de composição de alimentos. ${ }^{9}$ Para a análise estatística descritiva utilizou-se o programa Microsoft Office Excel, onde se tabularam também as informações em planilhas.

Para a coleta das informações, foi utilizada uma ficha de atendimento, composta por quatro partes. A primeira tratou da identificação do participante coletando: nome, sexo, endereço, cidade, telefone, data de nascimento, idade registrada no documento de identificação pessoal, estado civil, ocupação, renda e composição familiar. 
A segunda coletou dados de saúde como: ser fumante, ter apresentou alterações de peso, prática de atividades físicas, preferências alimentares, quem compra os alimentos e prepara as refeições, quais temperos são utilizados na preparação, frequência da compra, com quem realiza as refeições, a frequência de consumo de bebidas alcoólicas, se segue alguma dieta, o fracionamento das refeições, uso de medicamentos, consumo de água, gordura, açúcar e sal.

A terceira parte foi composta pela avaliação antropométrica e nutricional, coletando-se peso, altura, perímetro abdominal, índice de massa corporal (IMC), R24h e aferição da pressão arterial (PA). A quarta e última parte englobou os exames bioquímicos como glicemia de jejum e lipidograma, a partir da coleta de amostras sanguíneas, realizada por laboratório particular. O gasto energético total dos participantes teve como base a necessidade energética estimada Estimated Energy Requeriments (EER), conforme proposto pelo Institute of Medicine..$^{10}$

Para antropometria foram utilizados os seguintes equipamentos: balança de plataforma digital, com capacidade de $150 \mathrm{~kg}$ e sensibilidade de $100 \mathrm{~g}$, da marca Welmy; fita métrica inextensível e inelástica, de 2,0 m de extensão, com sensibilidade de 1,0 cm, da marca Stanley; estadiômetro de parede, da marca Sanny, com comprimento variando entre 80 e $220 \mathrm{~cm}$. A pressão arterial foi aferida durante o atendimento laboratorial com um esfigmomanômetro digital da marca Omron.

O peso foi aferido com o indivíduo posicionado na posição ortostática, em pé, sem sapatos e vestido apenas com "roupas leves", segundo o recomendado pelo Anthropometric Standardization Reference Manual. ${ }^{11}$

A altura foi aferida com a pessoa descalça, com os pés juntos e calcanhares encostados na parede, sem rodapé. O indivíduo foi posicionado em pé e ereto, sob uma superfície plana, com os braços soltos ao longo do corpo e as mãos voltadas para as coxas, a cabeça, escápula e glúteos foram todos alinhados paralelos à parede, com cabeça no plano de Frankfurt. ${ }^{12}$

O IMC determinou se o peso de um indivíduo estava adequado para a altura, estabelecendo o risco de mortalidade associado à presença de enfermidades, sendo estabelecido através de uma fórmula onde se tem o peso dividido pela altura ao quadrado $\left(\mathrm{IMC}=\right.$ peso $\left./(\text { altura })^{2}\right)$.

A classificação do estado nutricional foi realizada conforme recomendação da World Health Organization - $\mathrm{WHO}^{13}$ para adultos em: Magreza grau III - grave $\left(<16 \mathrm{~kg} / \mathrm{m}^{2}\right)$, Magreza grau II - moderada (16,0 a 16,9 kg/m²), Magreza grau I - leve (17,0 a $\left.18,4 \mathrm{~kg} / \mathrm{m}^{2}\right)$, Eutrofia (18,5 a 24,9 $\mathrm{kg} / \mathrm{m}^{2}$ ), Sobrepeso (25,0 a 29,9 kg/m²), Obesidade grau I ( 30 a $34,9 \mathrm{~kg} / \mathrm{m}^{2}$ ), Obesidade grau II (35 a $\left.39,9 \mathrm{~kg} / \mathrm{m}^{2}\right)$ e Obesidade grau III $\left(\geq 40 \mathrm{~kg} / \mathrm{m}^{2}\right)$.

A aferição da pressão arterial foi realizada no braço esquerdo, utilizando o esfigmomanômetro

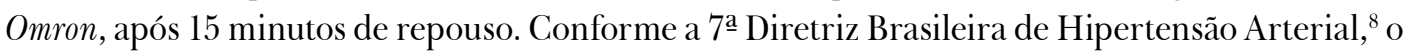


valor de referência para definição de Hipertensão Arterial Sistêmica (HAS) de PA sistólica adotado foi $\geq 140 \mathrm{mmHg}$ e/ou PA diastólica $\geq 90 \mathrm{mmHg}$.

O perímetro abdominal foi aferido com o paciente em pé, utilizando uma fita métrica não extensível, a partir do ponto médio, entre o rebordo costal e a crista ilíaca, como determina a World Health Organization. ${ }^{14} \mathrm{O}$ ponto de corte, de acordo com o NCEP, ${ }^{1}$ foi de $102 \mathrm{~cm}$ para homens e $88 \mathrm{~cm}$ para mulheres.

A avaliação da glicemia em jejum levou em conta o valor de referência que é menor que 99 $\mathrm{mg} / \mathrm{dL}$, segundo a Diretriz da Sociedade Brasileira de Diabetes. ${ }^{15}$ Para o lipidograma, realizado em jejum, os valores de referência para colesterol total, HDL-colesterol, triacilgliceróis (TG) e LDL-colesterol foram os propostos pela Atualização da Diretriz Brasileira de Dislipidemias e Prevenção da Aterosclerose 2017.16

Após a primeira consulta, em que foi realizado o diagnóstico nutricional, houve retorno com 5 pacientes dentre os 18 participantes, os quais apresentaram alterações nos exames bioquímicos tais como glicemia de jejum e lipidograma. Os mesmos receberam orientação para preencher três diários alimentares, em dias não consecutivos, incluindo um dia do final de semana, para posterior avaliação. Após análise dos diários, 4 pacientes receberam orientações nutricionais para perda de peso e dislipidemias e 1 recebeu prescrição dietética, com plano alimentar e lista de substituição.

\section{Resultados}

Na população estudada, 61,1\% eram do sexo feminino e 38,9\% eram do sexo masculino; $55,5 \%$ eram solteiros; $50 \%$ não faziam uso de bebidas alcoólicas; $66,6 \%$ não fumavam; $55,5 \%$ tinham como ocupação ser auxiliar de cozinha; possuíam idade média de 31,3 anos, composição familiar com média de 3,5 pessoas e renda média per capta de $\mathrm{R} \$ 605,55$.

Em relação às informações sobre o histórico alimentar dos participantes, pode-se observar que dentre os entrevistados, $(55,5 \%)$ realizavam as compras da casa sozinhos, com periodicidade mensal $(55,5 \%)$, preparavam suas próprias refeições $(55,5 \%)$ e comiam com a família e colegas de trabalho $(61,1 \%)$.

A média de refeições por dia foi de 3,2 vezes. Nas preparações, a grande maioria $(72,2 \%)$ fez uso de temperos industrializados e não utilizou sal nas refeições depois de prontas (38,8\%). A quantidade per capta consumida de açúcar foi de (3,25 Kg/mês) e óleo de soja (1,98L/mês).

No que se refere às preferências alimentares, o grupo das carnes foi o mais citado $(30,43 \%)$, seguido de leguminosas $(21,73 \%)$, cereais e hortaliças $(17,39 \%)$, frutas $(8,69 \%)$ e doces $(4,34 \%)$. 
No que concerne à prática de atividades físicas, observou-se que apenas 33,3\% as praticavam regularmente. Quanto ao uso de medicamentos, $44,4 \%$ faziam uso de pelo menos um, sendo que $25 \%$ relataram o uso de contraceptivos orais, $37,5 \%$ de anti-hipertensivos, $12,5 \%$ usavam hipoglicemiante, antidepressivo e corticoide.

O estado nutricional baseado no IMC, para participantes de ambos os sexos, está descrito na figura 1.

\section{Diagnóstico Nutricional}

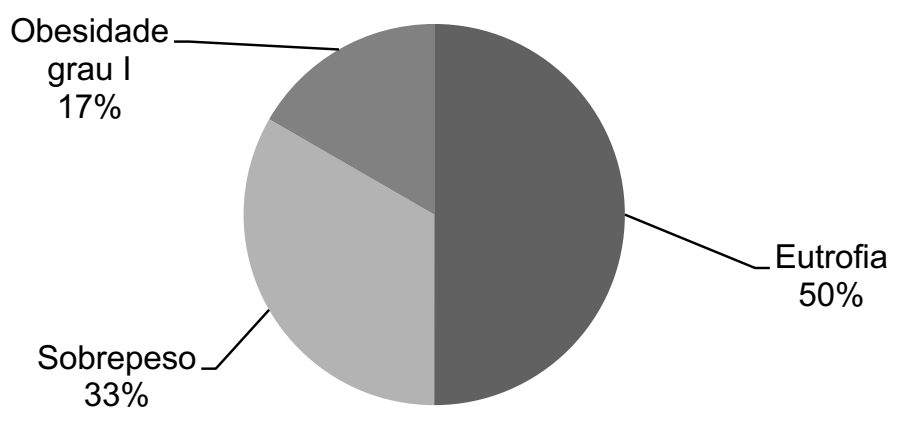

Figura 1. Diagnóstico nutricional dos funcionários do Restaurante Universitário da UFT, Palmas, 2016.

No que diz respeito ao perímetro abdominal, $27,2 \%$ das mulheres e $42,8 \%$ dos homens estavam acima dos valores considerados normais, indicando $33 \%$ da população estudada com maior risco para desenvolvimento de doenças crônicas como diabetes mellitus, hipertensão arterial sistêmica e doenças cardiovasculares. Dentre os valores de pressão arterial (PA), 55,5\% dos indivíduos pesquisados ultrapassaram apenas no valor da referência de PA sistólica, sem alterações na PA diastólica.

A tabela 1 mostra os percentuais de participantes conforme valores de referência para o lipidograma e glicemia de jejum. 
Tabela 1. Percentuais de participantes $(n=15)$ conforme os valores de referência para o lipidograma e glicemia de jejum. Palmas, TO, 2016.

\begin{tabular}{|c|c|}
\hline Dosagem Bioquímica & Participantes \\
\hline \multicolumn{2}{|l|}{ Glicemia de Jejum } \\
\hline$<99 \mathrm{mg} / \mathrm{dL}$ & $93,3 \%$ \\
\hline$\geq 99 \mathrm{mg} / \mathrm{dL}$ & $6,7 \%$ \\
\hline \multicolumn{2}{|l|}{ Colesterol Total } \\
\hline Desejável $(<190$ mg/dL) & $66,7 \%$ \\
\hline Alto $(\geq 190 \mathrm{mg} / \mathrm{dL})$ & $33,3 \%$ \\
\hline \multicolumn{2}{|l|}{ Triacilgliceróis } \\
\hline Desejável $(<150 \mathrm{mg} / \mathrm{dL})$ & $86,6 \%$ \\
\hline Limítrofe (150-199 mg/dL) & $6,7 \%$ \\
\hline Alto $(200-499 \mathrm{mg} / \mathrm{dL})$ & $6,7 \%$ \\
\hline Muito alto ( $\geq 500 \mathrm{mg} / \mathrm{dL})$ & 0 \\
\hline \multicolumn{2}{|l|}{ LDL - colesterol } \\
\hline Ótimo (< 100 mg/dL) & $40 \%$ \\
\hline Desejável (100-129 mg/dL) & $20 \%$ \\
\hline Limítrofe (130-159 mg/dL) & $13,3 \%$ \\
\hline Alto (160-189 mg/dL) & $26,7 \%$ \\
\hline Muito alto ( $\geq 190 \mathrm{mg} / \mathrm{dL})$ & 0 \\
\hline \multicolumn{2}{|l|}{ HDL - colesterol } \\
\hline Desejável (> 40 mg/dL) & $66,7 \%$ \\
\hline Baixo $(\leq 40 \mathrm{mg} / \mathrm{dL})$ & $33,3 \%$ \\
\hline
\end{tabular}

Observou-se que, apesar da maioria dos participantes estarem fora do risco cardiometabólico para as dosagens bioquímicas de glicemia e lipidograma, parcelas expressivas apresentaram colesterol total elevado (33,3\%), LDL-colesterol elevado (26,7\%) e baixo HDL-colesterol (33,3\%), o que eleva o risco cardiometabólico nesses participantes.

Os percentuais médios de ingestão diária de macronutrientes pelos 18 funcionários, baseados no inquérito alimentar aplicado, encontram-se na figura 2. O consumo de micronutrientes é mostrado na tabela 2 . 


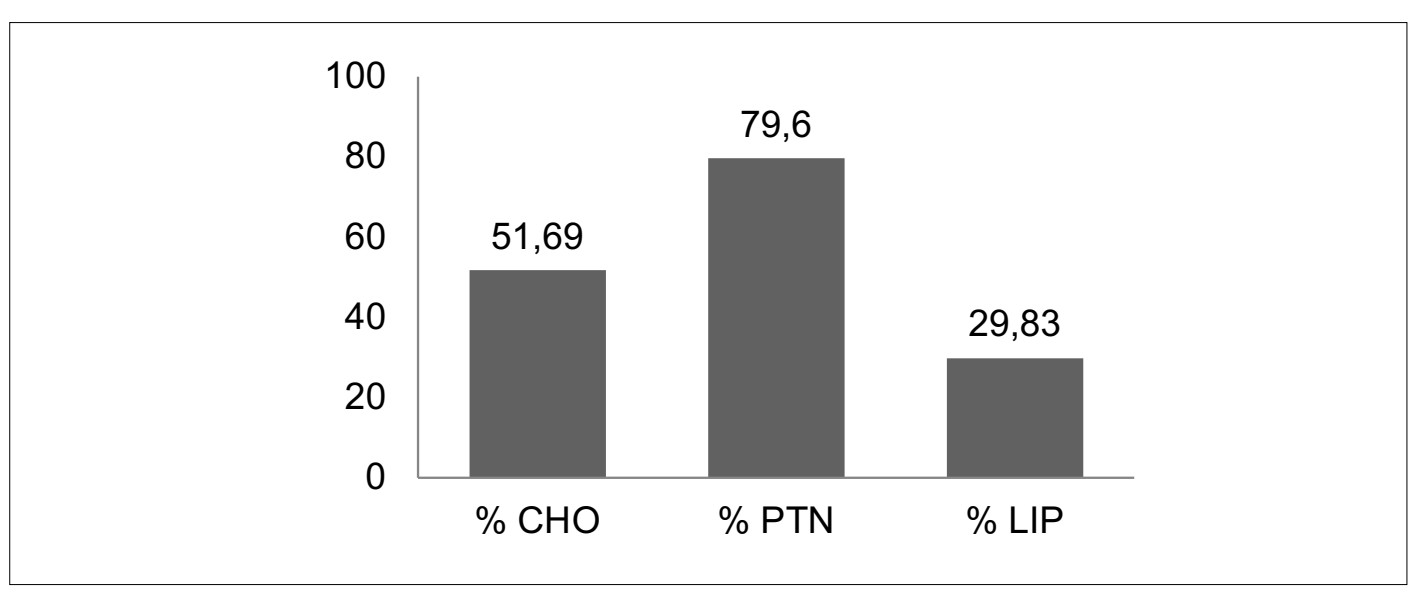

Figura 2. Percentuais médios de ingestão diária de macronutrientes pelos 18 funcionários do Restaurante Universitário da UFT, Palmas, 2016.

Tabela 2. Valores médios de ingestão diária de micronutrientes pelos 18 funcionários do Restaurante Universitário da UFT, Palmas,TO, 2016.

\begin{tabular}{|c|c|c|}
\hline Micronutrientes & Ingestão Média & Recomendações \\
\hline Colesterol (mg) & 206,48 & $<200 \mathrm{mg} / \mathrm{dia}^{* *}$ \\
\hline Fibras (g) & 19,26 & $30,6 \mathrm{~g} / \mathrm{dia}^{*}$ \\
\hline Gordura Monoinsaturada (g) & $\begin{array}{c}18,5 \\
9,97 \% \text { VET }\end{array}$ & $\leq 20 \% \mathrm{VET}^{* *}$ \\
\hline Gordura Trans (g) & 256,9 & $2 \mathrm{~g} / \mathrm{dia}^{* * *}$ \\
\hline Gordura Poliinsaturada (g) & $\begin{array}{c}7,76 \\
4,18 \% \text { VET }\end{array}$ & $\leq 10 \% \mathrm{VET}^{* *}$ \\
\hline Gordura Saturada (g) & $\begin{array}{c}1,90 \\
1,02 \% \mathrm{VET}\end{array}$ & $\leq 7 \% \mathrm{VET}^{* *}$ \\
\hline Cálcio (mg) & 339,21 & $1.142,86 \mathrm{mg} / \mathrm{dia}^{*}$ \\
\hline Ferro (mg) & 9,91 & $11,3 \mathrm{mg} / \mathrm{dia}^{*}$ \\
\hline Fósforo (mg) & 825,99 & $837,5 \mathrm{mg} / \mathrm{dia}^{*}$ \\
\hline Magnésio (mg) & 206,07 & $361,25 \mathrm{mg} / \mathrm{dia}^{*}$ \\
\hline Potássio (mg) & $1.913,54$ & $4.600 \mathrm{mg} / \mathrm{dia}^{*}$ \\
\hline Sódio (mg) & $1.452,35$ & $1.437,5 \mathrm{mg} / \mathrm{dia} *$ \\
\hline
\end{tabular}




\begin{tabular}{|c|c|c|}
\hline Micronutrientes & Ingestão Média & Recomendações \\
\hline Manganês (mg) & 2,29 & $2 \mathrm{mg} / \mathrm{dia}^{*}$ \\
\hline Selênio (mcg) & 55,79 & $53,12 \mathrm{mg} / \mathrm{dia}^{*}$ \\
\hline Zinco (mg) & 10,45 & $9,6 \mathrm{mg} / \mathrm{dia}^{*}$ \\
\hline Vit. A (mg) & 343,86 & $787,5 \mathrm{mcg} / \mathrm{dia} *$ \\
\hline Vit. C (mg) & 93,33 & $77,5 \mathrm{mg} / \mathrm{dia} *$ \\
\hline Vit. Bl (mg) & 1,03 & $1,11 \mathrm{mg} / \mathrm{dia}^{*}$ \\
\hline Vit. B2 (mg) & 0,91 & $1,16 \mathrm{mg} / \mathrm{dia}^{*}$ \\
\hline Vit. B3 (mg) & 10,46 & $14,75 \mathrm{mg} / \mathrm{dia}^{*}$ \\
\hline Vit. B6 (mg) & 1,11 & $1,35 \mathrm{mg} / \mathrm{dia}^{*}$ \\
\hline Vit. B9 (mcg) & 138,32 & $387,5 \mathrm{mcg} / \mathrm{dia} *$ \\
\hline Vit. B12 (mcg) & 3,67 & 2,32 mcg/dia* \\
\hline Vit. E (mg) & 6,05 & $14,5 \mathrm{mg} / \mathrm{dia}^{*}$ \\
\hline Vit. D (mcg) & 1,46 & $6,8 \mathrm{mcg} / \mathrm{dia}^{*}$ \\
\hline
\end{tabular}

VET = valor energético total da dieta

* Média de recomendações segundo a Dietary Reference Intakes (DRI), ${ }^{17}$ considerando homens e mulheres adultos e uma faixa etária de 19 a 50 anos.

**Recomendações segundo a IV Diretriz Brasileira sobre Dislipidemias e Prevenção da Aterosclerose (2007). ${ }^{18}$

****ecomendação segundo Anvisa. ${ }^{19}$

Destacam-se alguns nutrientes consumidos abaixo das recomendações como, por exemplo, das fibras, cálcio, ferro, fósforo, magnésio, potássio, vitamina A, vitamina B1 (tiamina), vitamina B2 (riboflavina), vitamina B3 (niacina), vitamina B6 (piridoxina), vitamina B9 (folato), vitamina E e vitamina D (tabela 2).

No entanto, os valores de ingestão de sódio, manganês, selênio, zinco, vitamina C e vitamina B12 (cobalamina) apresentaram-se superiores às recomendações (tabela 2).

Contudo, os valores das gorduras saturadas, monoinsaturadas e poliinsaturadas e colesterol obtidos nos resultados estavam dentro do recomendado, enquanto o valor da gordura trans, estava muito acima das recomendações (tabela 2).

Não obstante, em relação aos Diários Alimentares dos 5 participantes com alterações nos exames bioquímicos foi possível comparar os dados obtidos com as recomendações pelo Intervalo Aceitável de Distribuição dos Macronutrientes (Acceptable Macronutrient Distribuition Ranges - AMDR), proposto 
pelo Instituto de Medicina. ${ }^{20}$ Para o funcionário 1, o consumo de proteínas e lipídios estava acima das referências, uma vez que a recomendação é de $10 \%$ a $35 \%$ e $20 \%$ a $35 \%$, respectivamente. Já o consumo de carboidratos estava dentro do intervalo das recomendações de $45 \%$ a $65 \%$.

A tabela 3 mostra o consumo calórico obtido por meio dos R24h aplicados.

Tabela 3. Calorias diárias ingeridas obtidas através do R24h dos 5 funcionários do Restaurante Universitário da UFT, Palmas,TO, 2016.

\begin{tabular}{ccc}
\hline Funcionários & Calorias (Kcal) & EER (Kcal) \\
\hline 1 & $2.155,15$ & $2.112,5$ \\
2 & $1.322,68$ & $1.862,5$ \\
3 & $1.036,75$ & 1.730 \\
4 & $1.131,60$ & $1.622,5$ \\
5 & $1.511,41$ & $1.711,2$ \\
\hline
\end{tabular}

A média de calorias diárias ingeridas obtidas através do R24h foi de 1.669,40 Kcal, sendo que $38,8 \%$ consumiram a mais e $55,5 \%$ a menos do que o EER calculado.

As análises dos diários alimentares, realizados pelos cinco funcionários que apresentaram alterações nos exames bioquímicos são mostradas na figura 3 e tabela 4 .

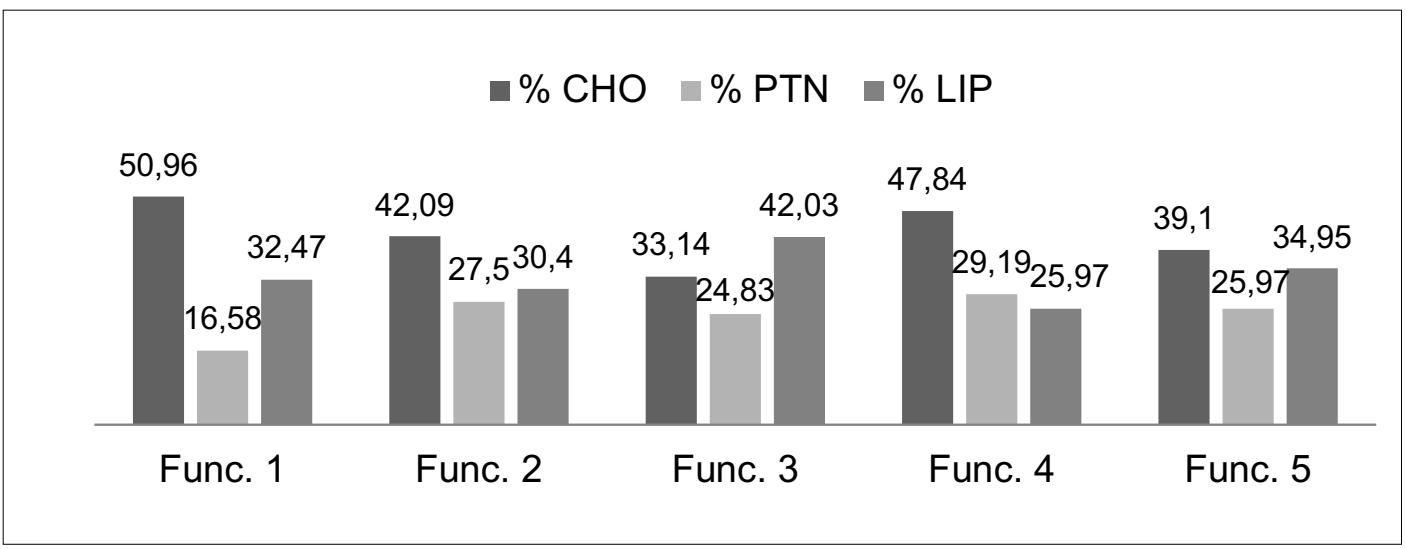

Figura 3. Percentuais médios de ingestão diária de macronutrientes pelos 5 funcionários do Restaurante Universitário da UFT, Palmas, TO 2016. 
Tabela 4. Ingestão média diária de micronutrientes, obtidas por registro alimentar de 3 dias, para funcionários do Restaurante Universitário da UFT, Palmas,TO, 2016.

\begin{tabular}{|c|c|c|c|c|c|c|}
\hline Micronutrientes & Func. 1 & Func. 2 & Func. 3 & Func. 4 & Func. 5 & Recomendação \\
\hline $\begin{array}{l}\text { Colesterol } \\
\text { (mg/dia) }\end{array}$ & 253,11 & 293,28 & 161,80 & 121,38 & 283,79 & $<200 \mathrm{mg} / \mathrm{dia}^{*}$ \\
\hline Fibras (g/dia) & 16,04 & 6,60 & 9,19 & 17,05 & 19 & $30,6 \mathrm{~g} / \mathrm{dia}^{* * *}$ \\
\hline Gordura & 23,02 & 12,9 & 15,82 & 11,38 & 22,17 & $\leq 20 \% \mathrm{VET}^{*}$ \\
\hline $\begin{array}{l}\text { Monoinsaturada } \\
\text { (g/dia) }\end{array}$ & $9,6 \% \mathrm{VET}$ & $8,7 \% \mathrm{VET}$ & $13,7 \% \mathrm{VET}$ & $9 \% \mathrm{VET}$ & $13,2 \% \mathrm{VET}$ & \\
\hline $\begin{array}{l}\text { Gordura Trans } \\
\text { (g/dia) }\end{array}$ & 3,77 & 0,44 & 0,60 & 0,28 & 0,11 & $2 \mathrm{~g} / \mathrm{dia}^{* *}$ \\
\hline Gordura & 10,47 & 7,92 & 5,98 & 1,54 & 10,03 & $\leq 10 \%$ VET $^{*}$ \\
\hline Poliinsaturada (g/dia) & $4,3 \% \mathrm{VET}$ & $5,38 \%$ VET & $5,19 \%$ VET & $1,22 \% \mathrm{VET}$ & $5,97 \%$ VET & \\
\hline $\begin{array}{l}\text { Gordura Saturada } \\
\text { (g/dia) }\end{array}$ & $\begin{array}{c}21,21 \\
8,80 \% \mathrm{VET}\end{array}$ & $\begin{array}{c}17,41 \\
11,8 \% \mathrm{VET}\end{array}$ & $\begin{array}{c}15,47 \\
13,4 \% \mathrm{VET}\end{array}$ & $\begin{array}{c}13,26 \\
10,5 \% \mathrm{VET}\end{array}$ & $\begin{array}{c}25,01 \\
14,9 \% \mathrm{VET}\end{array}$ & $\leq 7 \% \mathrm{VET}^{*}$ \\
\hline Cálcio (mg/dia) & 458,33 & 249,03 & 208,64 & 118,76 & 331,36 & $1.142,86 \mathrm{mg} / \mathrm{dia}^{* * *}$ \\
\hline Ferro (mg/dia) & 14,62 & 8,62 & 8,14 & 6,25 & 7,02 & $11,3 \mathrm{mg} / \mathrm{dia}{ }^{* * *}$ \\
\hline $\begin{array}{l}\text { Fósforo } \\
\text { (mg/dia) }\end{array}$ & 957,86 & 976,95 & 857,52 & 578,60 & 887,65 & $837,5 \mathrm{mg} / \mathrm{dia}^{* * *}$ \\
\hline $\begin{array}{l}\text { Magnésio } \\
\text { (mg/dia) }\end{array}$ & 198,66 & 204,80 & 124,61 & 118,81 & 151,3 & $361,25 \mathrm{mg} / \mathrm{dia}^{* * *}$ \\
\hline Potássio (mg/dia) & $2.204,25$ & $1.832,18$ & $1.271,99$ & $1.049,71$ & $1.567,35$ & $4.600 \mathrm{mg} / \mathrm{dia}^{* * *}$ \\
\hline Sódio (mg/dia) & $3.011,63$ & $1.029,22$ & 938,73 & $2.038,79$ & $2.192,63$ & $1.437,5 \mathrm{mg} / \mathrm{dia}^{* * *}$ \\
\hline Manganês (mg/dia) & 1,91 & 0,8 & 0,59 & 1,41 & 1 & $2 \mathrm{mg} / \mathrm{dia}^{* * *}$ \\
\hline Selênio (mg/dia) & 99,22 & 100,17 & 61,71 & 5,99 & 46,37 & $53,12 \mathrm{mg} / \mathrm{dia}^{* * *}$ \\
\hline Zinco (mg/dia) & 10,53 & 10,42 & 10,91 & 11,63 & 14,49 & $9,6 \mathrm{mg} / \mathrm{dia}^{* * *}$ \\
\hline Vit. A (mcg/dia) & 479,35 & 319,47 & 115,9 & 159,41 & 229,73 & $787,5 \mathrm{mcg} / \mathrm{dia}^{* * * *}$ \\
\hline Vit. C (mg/dia) & 156,7 & 18,45 & 18,24 & 12.05 & 216,55 & $77,5 \mathrm{mg} / \mathrm{dia}^{* * * *}$ \\
\hline Vit. B1 (mg/dia) & 1,66 & 0,71 & 0,67 & 0,29 & 0,76 & $1,11 \mathrm{mg} / \mathrm{dia}^{* * *}$ \\
\hline Vit. B2 (mg/dia) & 1,24 & 0,99 & 0,84 & 0,17 & 0,81 & $1,16 \mathrm{mg} / \mathrm{dia}{ }^{* * *}$ \\
\hline Vit. B3 (mg/dia) & 18,39 & 12,67 & 10,17 & 6,77 & 13,92 & $14,75 \mathrm{mg} / \mathrm{dia}^{* * * *}$ \\
\hline
\end{tabular}




\begin{tabular}{lcccccc}
\hline Micronutrientes & Func. 1 & Func. 2 & Func. 3 & Func. 4 & Func. 5 & Recomendação \\
\hline Vit. B6 (mg/dia) & 1,49 & 1,17 & 1,14 & 0,20 & 0,83 & $1,35 \mathrm{mg} / \mathrm{dia}$ *** \\
Vit. B9 (mcg/dia) & 235,58 & 115,23 & 56,94 & 27,72 & 40,62 & $387,5 \mathrm{mcg} / \mathrm{dia}$ *** \\
Vit. B12 (mcg/dia) & 7,84 & 6,8 & 4,82 & 0,18 & 0,79 & $2,32 \mathrm{mcg} / \mathrm{dia} * * *$ \\
Vit. E (mg/dia) & 10,21 & 5,15 & 5,59 & 0,38 & 3,71 & $14,5 \mathrm{mg} / \mathrm{dia} * * *$ \\
Vit. D (mcg/dia) & 4,60 & 2,44 & 1,23 & 0,43 & 1,04 & $6,8 \mathrm{mcg} / \mathrm{dia} * * *$ \\
\hline
\end{tabular}

Func.= Funcionário. VET $=$ valor energético total da dieta

*Recomendações segundo a IV Diretriz Brasileira sobre Dislipidemias e Prevenção da Aterosclerose (2007). ${ }^{18}$

**Recomendação segundo Anvisa. ${ }^{19}$

***Média de recomendações segundo a Dietary Reference Intakes (DRI), ${ }^{17}$ considerando homens e mulheres adultos e uma faixa etária de 19 a 50 anos.

Quanto aos micronutrientes, os valores de alguns encontraram-se abaixo das recomendações, como por exemplo, as fibras, cálcio, magnésio, manganês, potássio, vitamina A, vitamina B9 (folato), vitamina E e vitamina D. Além disso, alguns micronutrientes apresentaram valores acima das recomendações, tais como o ferro, fósforo, sódio, selênio, zinco, vitamina C, vitamina B1 (tiamina), vitamina B2 (riboflavina), vitamina B3 (niacina), vitamina B6 (piridoxina) e vitamina B12 (tabela 4).

No entanto, os valores das gorduras saturadas, trans e do colesterol encontraram-se acima do recomendado, enquanto as gorduras monoinsaturadas e poliinsaturadas encontraram-se dentro das referências (tabela 4).

Para os funcionários 2 e 3, no que se refere aos dados dos macronutrientes, os valores de ingestão de proteínas e lipídios estão acima das referências, uma vez que a recomendação é de $10 \%$ a $35 \%$ e $20 \%$ a 35\%, respectivamente. Com relação ao consumo de carboidratos, o funcionário 2apresenta-se dentro das recomendações de $45 \%$ a $65 \%$, enquanto ofuncionário 3, estava abaixo das recomendações (figura 3).

Quanto aos micronutrientes dos funcionários 2 e 3 foi notório que alguns estavam abaixo das recomendações, como por exemplo, as fibras, cálcio, ferro, magnésio, manganês, potássio, sódio, vitamina A, vitamina C, vitamina B1 (tiamina), vitamina B2 (riboflavina), da vitamina B3 (niacina), da vitamina B6 (piridoxina), vitamina B9 (folato), vitamina E e da vitamina D (tabela 4).

Todavia, alguns micronutrientes encontraram-se com os valores acima das recomendações, tais como fósforo, selênio, zinco e vitamina B12 (tabela 4). Além disso, a ingestão de colesterol do funcionário 1 estava acima das recomendações, enquanto que a do funcionário 2, estava dentro do recomendado. E, por fim, no que se diz respeito ao consumo de gorduras dos funcionários 1 e 2, o consumo de saturadas estavam acima da recomendação, enquanto os de monoinsaturadas, poliinsaturadas e trans estavam dentro do recomendado (tabela 4). 
Contudo, os funcionários 4 e 5 apresentaram alterações na ingestão de macronutrientes, sendo que o consumo de proteínas se apresentou acima dos valores de referência (10\% a 35\%). Em relação aos lipídios, o consumo do funcionário 4 estava dentro das recomendações (20\% a 35\%), mas o consumo do funcionário 5 estava acima do recomendado. Com relação ao consumo de carboidratos, o funcionário 4 apresentou ingestão dentro do intervalo das recomendações (45\% a $65 \%$ ), mas o funcionário 5, apresentou ingestão acima do recomendado (figura 3).

Quanto à ingestão de micronutrientes, as fibras, cálcio, ferro, magnésio, manganês, potássio, selênio, vitamina A, vitamina B1 (tiamina), vitamina B2 (riboflavina), vitamina B3 (niacina), vitamina B6 (piridoxina), vitamina B9 (folato), vitamina B12, vitamina $\mathrm{E}$ e vitamina $\mathrm{D}$ estavam abaixo das recomendações. Já a vitamina $\mathrm{C}$ do funcionário 4 estava abaixo das recomendações, ao contrário do funcionário 5 , que se encontrava acima do recomendado (tabela 4).

Apesar disso, alguns micronutrientes estavam acima das recomendações, tais como o sódio e o zinco. Quanto ao fósforo, este obteve valores de ingestão abaixo das recomendações para o funcionário 4, e acima para o funcionário 5. Ademais, a ingestão de colesterol dos funcionários 4 e 5 superou o recomendado e, por fim, no que se refere às gorduras, as saturadas foram ingeridas acima da recomendação e as ingestões das monoinsaturadas, poliinsaturadas e trans, mantiveramse dentro do recomendado (tabela 4).

\section{Discussão}

Os dados antropométricos obtidos no presente estudo revelam percentuais consideráveis de sobrepeso (33\%) e obesidade (17\%). Em estudos realizados no Brasil tem sido observado aumento na prevalência de sobrepeso e obesidade. Neste trabalho Tonini et al. ${ }^{21}$ foram avaliados o estado nutricional e o hábito alimentar dos funcionários de uma instituição de ensino superior do oeste de Santa Catarina, onde 39,3\% dos funcionários apresentavam excesso de peso.

Do mesmo modo, Simon et al. ${ }^{22}$ encontraram $60,8 \%$ dos profissionais do serviço de nutrição e dietética de um hospital de Porto Alegre com excesso de peso. Quanto aos exames bioquímicos, o autor observou alteração da glicemia de jejum, LDL-C alto e HDL-C baixo. De maneira semelhante ao que foi encontrado em nossos resultados onde 11,1\% apresentaram elevados níveis de glicemia de jejum, aumento de LDL-c (>16,6\%) e HDL-c abaixo do desejável (<33,3\%), o que reflete um perfil de hipercolesterolemia e grande risco de desenvolvimento de doenças cardiovasculares, como observado também por Ruiz-Fernandez et al. ${ }^{23}$ que avaliaram RC em uma comunidade localizada na Venezuela.

No estudo de Mariath et al. ${ }^{24} 33 \%$ dos colaboradores de uma indústria de Santa Catarina foram identificados com perímetro abdominal elevado, similar ao encontrado no presente estudo (33\%), o que representa maior risco para o desenvolvimento de doenças metabólicas associadas à obesidade. 
Nos R24h analisados, a média de consumo energético diário foi de 1.669,40 Kcal, mas a maioria dos participantes $(55,5 \%)$ ingeriu menos do que o recomendado pela EER calculada. São 330,6Kcal a menos relativo ao valor calórico do funcionário adulto proposto pelo Programa de Alimentação do Trabalhador (PAT), com valor aproximado de $2.000 \mathrm{Kcal}$, distribuídas em desjejum, almoço, lanche, jantar e ceia. ${ }^{25}$

Ao compararmos o consumo de macronutrientes às recomendações pelo AMDR, proposto pelo IOM, ${ }^{20}$ nos resultados obtidos para o consumo de proteínas $(79,6 \%)$ mostram uma ingestão proteica acima da recomendação de $10 \%$ a $35 \%$ do valor energético total (VET) que deve ser ingerido diariamente. Quanto ao consumo de carboidratos, nota-se adequação nas recomendações, em que o valor médio de consumo de carboidratos estava em 51,6\%, já que recomendação é de $45 \%$ a 65\%. Para os lipídios, foi observado consumo médio na população estudada de 29,8\% do VET, sendo recomendados valores de ingestão entre $20 \%$ e $35 \%$.

Quanto aos micronutrientes, observou-se que o consumo médio de selênio dos participantes esteve acima do recomendado (55,79 mcg) pela Ingestão Dietética Recomendada (RDA), mas não ultrapassou o Nível Máximo de Ingestão Tolerável (UL). ${ }^{20}$ Nesse contexto, Stranges et al. ${ }^{26}$ analisaram as quantidades ingeridas de selênio em excesso e o risco de doenças cardiovasculares, mas não encontrou evidências nesta correlação.

Na população estudada também foi identificado que as recomendações de ingestão de fibras $(19,26 \mathrm{~g})$ não foram alcançadas e o consumo de sódio excedeu o recomendado, ${ }^{20}$ fatores que elevam os riscos cardiometabólicos. ${ }^{27}$ Esses mesmos autores constataram que dentre os riscos metabólicos em adultos coreanos, destacam-se a baixa ingestão de grãos integrais e frutas; a alta ingestão de sódio; além de parâmetros antropométricos desfavoráveis como índice de massa corporal e PA elevados, situação que também foi constatada no presente estudo onde elevações de PA prevaleceram em $55,5 \%$ dos indivíduos.

Da mesma maneira, foi possível constatar valores de ingestão de magnésio abaixo de recomendações (206,07 mg), o que leva a desproteção contra eventos cardiovasculares por reduzir a rigidez arterial. ${ }^{28}$ Esses mesmos autores investigaram adultos com obesidade e sobrepeso, suplementados com $350 \mathrm{mg} /$ dia de magnésio por 24 semanas e perceberam que houve impacto cardiovascular benéfico. Com relação à baixa ingestão de vitamina E observada no presente estudo, Hu et al. ${ }^{29}$ demonstraram efeito cardioprotetor da vitamina E, porém com mecanismos ainda desconhecidos, funcionando apenas em alguns pacientes e podendo ser afetado por fatores como idade e gênero.

A maioria dos participantes do presente estudo atingiu a recomendação de vitamina $\mathrm{C}$, com ingestão média de $93,33 \mathrm{mg}$ de vitamina C no grupo estudado. Soriano-Maldonado et al. ${ }^{30}$ postularam que o consumo conjunto de antioxidantes naturais do suco de maçã, como a vitamina 
C e os polifenóis podem ter efeitos favoráveis nos marcadores cardiometabólicos. Catania et al. ${ }^{31}$ também descreveram que estudos epidemiológicos propõem que dietas ricas em antioxidantes, ou simplesmente ricas em frutas e vegetais, podem reduzir RC.

No que diz respeito ao consumo de lipídios, chama atenção na população estudada, o consumo elevado de gordura trans, ou seja, acima de $2 \mathrm{~g} / \mathrm{dia}$, principalmente oriunda de alimentos industrializados, caracterizando um panorama desfavorável em relação aos RC. Em contrapartida, observou-se adequação no consumo de ácidos graxos monoinsaturados e poliinsaturados.

Oliveira et al. ${ }^{32}$ verificou que a introdução ou substituição de gorduras saturadas e trans por ácidos graxos insaturados trouxe benefícios para a redução do risco cardiovascular, em idosos obesos ou com excesso de peso e não diabéticos, conferindo, portanto, um fator de proteção.

\section{Conclusão}

O referido estudo retrata um panorama antropométrico e dietético desfavorável com evidencia de fatores que predispõem a população a eventos cardiovasculares, como o consumo elevado de gorduras trans, o baixo consumo de fibras, o sedentarismo e os hábitos de vida não saudáveis, a baixa ingestão de micronutrientes essenciais à homeostase e proteção do organismo, além da má distribuição dos macronutrientes da dieta.

O elevado índice de excesso de peso e obesidade da população analisada foi possivelmente causado por alterações dos padrões alimentares que envolvem tanto o consumo de industrializados, ricos em gorduras e açúcares, quanto à reduzida prática de atividade física. Assim sendo, o nutricionista é responsável por avaliar cada indivíduo, de forma holística, a fim de orientá-lo de acordo com suas necessidades e, dessa maneira, melhorar os parâmetros bioquímicos e nutricionais.

\section{Agradecimentos}

Aos participantes que colaboraram com este estudo.

\section{Colaboradores}

Todos os autores contribuíram em todas as etapas desde a concepção do estudo até a revisão da versão final do artigo.

Conflito de Interesses: Os autores declaram não haver conflito de interesses. 


\section{Referências}

1. National Cholesterol Education Program. Third report of the national cholesterol education program expert panel on detection, evaluation, and treatment of high blood cholesterol in adults (Adult Treatment Panel III) [Internet]. National Institutes of Health; 2001. [acesso em: 04 fev. 2016]. Disponível em: http://www.scymed.com/en/smnxdj/edzr/edzr9610.htm

2. Associação Brasileira para o Estudo da Obesidade e da Síndrome Metabólica. Diretrizes brasileiras de obesidade 2016. 4. ed. São Paulo: ABESO; 2016.

3. Pereira IVNFS. Síndrome Metabólica e risco cardiovascular [Dissertação]. [Coimbra]: Faculdade de Farmácia da Universidade de Coimbra, 2010.

4. Haab RS, Benvegnú LA, Fischer EV. Prevalência de Síndrome Metabólica em uma área rural de Santa Rosa. Rev Bras Med Fam Comunidade. 2012; 7(23):90-99.

5. Baxter AJ, Coyne T, McClintock C. Dietary patterns and Metabolic Syndrome: a review of epidemiologic evidence. Asia Pac J Clin Nutr. 2006; 15(2):134-142.

6. Castanho GKF, Marsola FC, Mclellan KCP, Nicola M, Moreto F, Burini RC. Consumo de frutas, verduras e legumes associado à Síndrome Metabólica e seus componentes em amostra populacional adulta. Ciênc. Saúde Coletiva. 2013; 18(2):385-392.

7. Busnello FM, Bodanese LC, Pellanda LC, Santos ZEA. Intervenção nutricional e o impacto na adesão ao tratamento em pacientes com Síndrome Metabólica. Arq Bras Cardiol. 2011; 97(3):217-224.

8. Sociedade Brasileira de Cardiologia. $7^{a}$ Diretriz brasileira de Hipertensão Arterial. Arq Bras Cardiol. [Internet]. 2016; 107(Supl. 3). Disponível em: http://publicacoes.cardiol.br/2014/diretrizes/2016/05_ HIPERTENSAO_ARTERIAL.pdf

9. Universidade Estadual de Campinas. Núcleo de Estudos e Pesquisas em Alimentação. Tabela Brasileira de Composição de Alimentos (TACO). $4^{a}$ ed. Campinas: NEPA - UNICAMP; 2011. 161 p.

10. Institute of Medicine. Dietary reference intakes: applications in dietary planning. Washington, DC: National Academies Press; 2002.

11. Gordon CC, Chumlea WC, Roche AF. Stature, recumbent length, and weight. In: Lohman TG, Roche AF, Martorell R. Editor. Anthropometric standardization reference manual. Illinois: Human Kinetics Books; 1991. p. 3-9.

12. Instituto Brasileiro de Geografia e Estatística. Manual de Antropometria. Laboratório de Avaliação Nutricional de Populações - LANPOP. Rio de Janeiro: IBGE; 2013.

13. World Health Organization. Obesity. Preventing and managing the global epidemic. Report of a WHO consultation on obesity. Geneva: World Health Organization; 1997. p. 107-158.

14. Organização Mundial de Saúde. Physical status: the use and interpretation of anthropometry. Geneva: WHO; 1995.

15. Oliveira JEP, Montenegro Junior RM, Vencio S. organizadores. Diretrizes da Sociedade Brasileira de Diabetes 2017-2018. São Paulo: Clannad; 2017. 
16. Sociedade Brasileira de Cardiologia. Atualização da diretriz brasileira de dislipidemias e prevenção da aterosclerose. Arq Bras Cardiol. 2017; 109(2):Sup.1.

17. Padovani RM, Amaya-Farfán J, Colugnati FAB, Domene SMA. Dietary reference intakes: aplicabilidade das tabelas em estudos nutricionais. Rev. Nutr. 2006; 19(6):741-760.

18. Sociedade Brasileira de Cardiologia. IV Diretriz brasileira sobre dislipidemias e prevenção da Aterosclerose. Arq Bras Cardiol. [Internet]. 2007; 88(Supl. 1). Disponível em: http://www.scielo.br/ pdf/abc/v88s1/01.pdf

19. Agência Nacional de Vigilância Sanitária. Informações técnicas sobre a gordura trans [Internet]. Portal da Anvisa. [acesso em: 16 maio 2017]. Disponível em: http://portal.anvisa.gov.br/ convenios?p_p_id=110_INSTANCE_lrSCVWqtmttC\&p_p_lifecycle=0\&p_p_state=normal\&p_p_ mode $=$ view\&_110_INSTANCE_lrSCVWqtmttC_struts_action=\%2Fdocument_library_ display $\% 2$ Fview_file_entry\&_110_INSTANCE_lrSCVWqtmttC_redirect=http $\% 3 \mathrm{~A} \% 2 \mathrm{~F} \% 2 \mathrm{Fportal}$. anvisa.gov.br $\% 2$ Fresultado-de-busca $\% 3 \mathrm{Fp}$-P_id $\% 3 \mathrm{D} 3 \% 26 \mathrm{p}$-P_lifecycle $\% 3 \mathrm{D} 0 \% 26 \mathrm{p}$-P_ state $\% 3$ Dnormal $\% 26$ p_p_mode $\% 3 D$ view $\% 26$ p_p_col_id $\% 3$ Dcolumn-1\%26p_p_col_

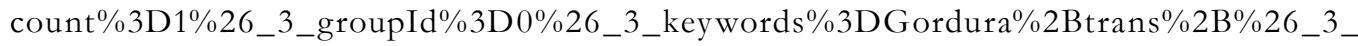
cur\%3D1\%26_3_struts_action $\% 3 \mathrm{D} \% 252 \mathrm{Fsearch} \% 252 \mathrm{Fsearch} \% 26$ _3_format $\% 3 \mathrm{D} \% 26$ _3_ formDate\%3D1441824476958\&_110_INSTANCE_lrSCVWqtmttC_fileEntryId=2695789

20. Institute of Medicine. Dietary reference intakes. Standing committee on the scientific evaluation of dietary reference intakes. Dietary reference intakes for energy, carbohydrate, fiber, fat, fatty acids, cholesterol, protein, and amino acids (Macronutrients) [Internet]. Washington, DC: The National Academies Press. 2005. 1357 p. [acesso em: 26 abr. 2017]. Disponível em: http://www.nap.edu/ catalog/10490.html

21. Tonini E, Broll AM, Corrêa EN. Avaliação do estado nutricional e hábito alimentar de funcionários de uma instituição de ensino superior do oeste de Santa Catarina. O Mundo da Saúde. 2013; 37(3):268-279.

22. Simon MISS, Garcia CA, Lino ND, Forte GC, Fontoura ID, Oliveira ABA. Avaliação nutricional dos profissionais do serviço de nutrição e dietética de um hospital terciário de Porto Alegre. Cad Saúde Coletiva. 2014; 22(1):69-74.

23. Ruiz-Fernández N, Espinoza M, Barrios E, Reigosa A. Factores Cardiometabólicos en una Comunidad de Valencia, Venezuela. Rev. Salud Pública. 2009; 11(3):383-394.

24. Mariath AB, Grillo LP, Silva RO, Schmitz P, Campos IC, Medina JRP, et al. Obesidade e fatores de risco para o desenvolvimento de doenças crônicas não transmissíveis entre usuários de unidade de alimentação e nutrição. Cad. Saúde Pública. 2007; 23(4):897-905.

25. Brasil. Ministério de Estado do Trabalho e Emprego, da Fazenda, da Saúde, da Previdência Social e do Desenvolvimento Social e Combate á Fome. Portaria interministerial no 66, de 25 de agosto de 2006. Altera os parâmetros nutricionais do Programa de Alimentação do Trabalhador - PAT [Internet]. [acesso em: 11 maio 2017. Disponível em: http://189.28.128.100/nutricao/docs/legislacao/ portaria66_25_08_06.pdfPAT

26. Stranges S, Galletti F, Farinaro E, D’elia L. Associations of selenium status with cardiometabolic risk factors: an 8-year follow-up analysis of the Olivetti Heart study. Atherosclerosis. 2011; 217(1):274-278. 
27. Cho Y, Cudhea F, Park JH, Mozaffarian D, Singh G, Shin MJ. Burdens of Cardiometabolic Diseases Attributable to Dietary and Metabolic Risks in Korean Adults 2012-2013. Yonsei Med J. 2017; 58(3):540-551.

28. Joris PJ, Plat J, Bakker SJ, Mensink RP. Long-term magnesium supplementation improves arterial stiffness in overweight and obese adults: results of a randomized, double-blind, placebo-controlled intervention trial. Am J Clin Nutr. 2016; 103(5):1260-1266.

29. Hu XX, Fu L, Li Y, Lin ZB, Liu X, Wang JF, et al. The cardioprotective effect of Vitamin E (AlphaTocopherol) is strongly related to age and gender in mice. PLoS One, 2015; 10(9): e0137405.

30. Soriano-Maldonado A, Hidalgo M, Arteaga P, Pascual-Teresa S, Nova E. Effects of regular consumption of Vitamin C-rich or polyphenol-rich apple juice on cardiometabolic markers in healthy adults: a randomized crossover trial. Eur J Nutr. 2014; 53(8):1645-1657.

31. Catania AS, Barros CR, Ferreira SR. Vitamins and minerals with antioxidant properties and cardiometabolic risk: controversies and perspectives. Arq Bras Endocrinol Metabol. 2009; 53(5):550-559.

32. Oliveira PA, Kovacs C, Moreira P, Magnoni D, Saleh MH, Faintuch J. Unsaturated fatty acids improve atherosclerosis markers in obese and overweight non-diabetic elderly patients. Obes Surg. 2017; 27(10):2663-2671.

Recebido: 04 de dezembro de 2017

Revisado: 21 de maio de 2018

Aceito: 29 de maio de 2018 